\title{
Are signaling pathways inversely regulated in Alzheimer's disease and glioblastoma multiforme?
}

\author{
Authors: \\ MS1: William G O'Neal, \\ Mentor: Debomoy K. Lahiri, Ph.D. \\ Clinical mentor: Mahua Dey, M.D. \\ Graduate student: Ruizhi Wang \\ (Other member of the lab: Bryan Maloney and Mario Henriquez) \\ Affiliation: \\ Departments of Psychiatry, Neurological Surgery, and Medical and Molecular \\ Genetics, Indiana University School of Medicine, Indiana Alzheimer Disease \\ Center (IADC), Stark Neuroscience Research Center, 320 West 15th Street, NB \\ 200, \\ Indianapolis, IN 46202, USA
}

Background: Epidemiological studies suggest an inverse association between cancer and neurodegenerative disorders, including Alzheimer's disease (AD). AD and cancers, such as glioblastoma multiforme (GBM), are characterized by abnormal but opposing cellular behavior. $A D$ is characterized by accumulation of the processing products of amyloid $\beta(A \beta)$ and its metabolizing enzymes amyloid precursor protein (APP), $\beta$-secretase (or BACE1), and $\gamma$-secretase. Our rationale is unraveling cell signaling pathways linking $A D$ and GBM. We hypothesized lowgrade gliomas (LGG) and high-grade gliomas (HGG) would have differential expression of neuronal and synaptic markers. Furthermore, protein expression profiles of these markers, APP metabolites, and BACE1 would be different among LGG, HGG, and AD cases.

Experimental Design or project methods: Specific neuronal protein markers (e.g., NSE), presynaptic proteins (e.g., synaptophysin and SNAP25), and postsynaptic proteins (e.g., PSD-95) have been measured in glioma samples. Characterization are done by Western immunoblotting and ELISA. Protein biomarkers will be analyzed in LGG and HGG of biopsy samples, and the results will be compared with brain samples from AD cases.

Results: Using specific primary and secondary antibodies and optimal protein range, we have standardized an immunoblotting procedure to detect our desired proteins in blinded LGG and HGG samples. After unblinding and analyzing results, expression signals will be compared between GBM and AD brain samples.

Conclusion and potential impact: Our results would shed light on diverging and/or shared cell signaling pathways between AD and GBM. In addition, potential impact would be utilizing GBM-derived cultures to test and develop therapeutics for both $A D$ and GBM. 